\title{
SLC2A4 gene: a promising target for pharmacogenomics of insulin resistance
}

\section{"...pharmacogenomics has great potential to select subjects more or less prone to regulate the SLC2A4 gene and consequently glycemic homeostasis."}

\section{KEYWORDS: diabetes $\approx$ GLUT4 = insulin resistance " pharmacogenomics $\approx$ SLC2A4}

\section{The SLC2A4 gene}

The GLUT4 protein, encoded by the solute carrier SLC2A4 gene in humans and Slc2a4 in mice and rats, is preferentially expressed in differentiated myotubes and adipocytes. Described in the early 1990s, GLUT4 is considered the insulin-sensitive glucose transporter based on its particular characteristic of being inserted in membranes of intracellular vesicles, which under insulin stimulus translocate to the plasma membrane, increasing the glucose uptake by these cells; the basis of postprandial glycemic control.

Insulin-induced GLUT4 translocation is described as a much more robust phenomenon than it really is in vivo. In vitro translocation compares maximal insulin effect with the socalled basal condition in which insulin is absent. Whenever one tries to compare maximal insulin effect with basal physiological insulin concentrations, GLUT4 translocation is very low [1]. Besides, in skeletal muscle, GLUT4 translocation is also stimulated by muscle contraction, and contractile tonus is enough to induce great levels of translocation. However, a small relative translocation of GLUT4 (e.g., small percentage related to the total content) is able to significantly increase glucose uptake, avoiding impaired postprandial hyperglycemia. Unbelievably, several studies concerning GLUT4 translocation simply analyze the absolute amount of GLUT4 in plasma membrane. We point out that insulin induces vesicle translocation; and reduction in SLC2A4 expression (decreasing GLUT4 density in the vesicles) can explain decreased insulin-stimulated plasma membrane GLUT4 content, despite a preserved translocation system [2]. Finally, although some studies have proposed that molecular changes in the GLUT4 protein might alter its kinetics of transport, no consistent data has confirmed this hypothesis $[3,4]$.
Finally, SLC2A4/GLUT4 expression seems to be the main element in tissue glucose disposal; and, consequently, in glycemic homeostasis [5]. Considering that, investigations should focus on the regulation of SLC2A4/GLUT4 expression.

\section{SLC2A4 expression \& insulin resistance}

To begin with, it is important to ponder that insulin resistance (IR) can be defined as and analyzed according to different points of view, without considering liver involvement. First, IR can be characterized in cells by detecting any impairment in the insulin signaling pathway, which not obligatorily results in impaired glucose uptake; adjustments in SLC2A4 expression and GLUT4 translocation can preserve normal glucose transport. Second, when SLC2A4/GLUT4 expression and insulin-induced glucose uptake are reduced in muscle and/or adipose cells, this does not guarantee that the plasma glucose clearance will be decreased; increased cellularity and/or tissue mass can counterbalance the cellular glucose disposal deficit. Finally, to infer about IR and impaired glycemic homeostasis, we need to analyze whole-body IR, defined by reduced insulin-induced plasma glucose clearance, for which total tissue GLUT4 content and consequently glucose disposal must be analyzed [6]. In non-obese subjects, skeletal muscle is the most important territory for insulin-induced glucose clearance; however, in obese subjects, adipose tissue can also become relevant.

Remarkably, decreased insulin-induced glucose uptake, associated with impaired insulin signaling pathways, have been currently related only to impaired GLUT4 translocation; however, most studies in this context fail to demonstrate that decreased SLC2A4/GLUT4 expression is not concomitantly involved. Currently, these studies analyze only the absolute

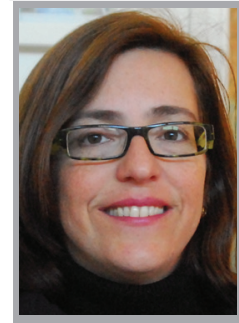

Maria Lucia Corrêa-Giannella Department of Internal Medicine, Endocrinology \& Laboratory for Cellular \& Molecular Endocrinology (LIM-25), School of Medicine, University of São Paulo, São Paulo, Brazil

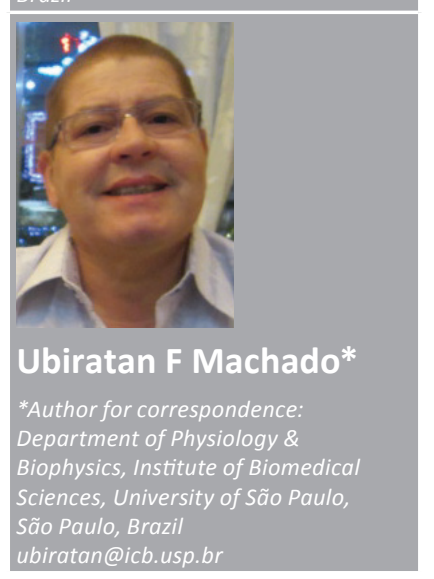

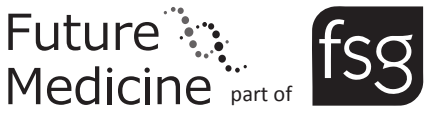


amount of plasma membrane GLUT4, without considering the total cell/tissue GLUT4 content. Thus, to understand the participation of SLC2A4/GLUT4 in cell/tissue/whole-body insulin-induced glucose clearance, different types of data analysis must be conducted [6].

\section{SLC2A4 expression in obesity $\&$ diabetes}

As soon as the SLC2A4 gene was characterized, SLC2A4 mRNA and GLUT4 protein content were analyzed in obese and diabetic subjects, and controversial results were described. For instance, in obese IR subjects, SLC2A4 expression in adipose tissue was described as increased, unchanged and decreased. In experimental models of severely obese IR animals, GLUT4 was reduced in all insulin sensitive tissues [7], and treatments that improve glycemic homeostasis increased GLUT4 content. These data depict the important role of SLC2A4 in the pathophysiology of obesity and diabetes.

\section{"...SLC2A4/GLUT4 expression seems to be the main element in tissue glucose disposal..."}

Interestingly, studying the development of obesity and diabetes mellitus (DM) in monosodium-glutamate-treated mice, we observed in adipose tissue that, earlier, when fat gain mass is accelerated, Slc2a 4 expression increases; however, later on, when progression of obesity stabilizes and severe IR is established, Slc2a4 expression decreases [8]. This pattern of Slc2a4 expression seems to be related to the inflammatory-induced NF- $\kappa \mathrm{B}$ activity, a repressor of the Slc2a 4 gene, and can explain the varied results of SLC2A4/GLUT4 expression reported in patients with obesity and Type 2 DM (T2DM).

Additionally, several controversial data concerning the SLC2A4 gene and glycemic homeostasis were based only on SLC2A4 mRNA quantification, without GLUT4 protein analysis. However, we and others have reported discrepancies between regulation of Slc $2 a 4 \mathrm{mRNA}$ and GLUT4 protein. Data suggest that changes in Slc2a 4 mRNA poly-A tail size, and in some specific miRNAs are involved in the posttranscriptional modulation of SLC2A4 expression, and investigations in this field should be strengthened.

Nevertheless, nowadays it is well accepted that IR will culminate in decreased SLC2A4 expression, not only in obesity and T2DM, but also in pregnancy, gestational diabetes, polycystic ovary syndrome, some types of hypertension, the so-called metabolic syndrome and so on.
Furthermore, although IR is not involved in the etiopathogeny of Type $1 \mathrm{DM}$, now we know that IR may also be present in such conditions; we have demonstrated in Type 1 DM rats that IR is accompanied by reduced GLUT4 content in skeletal muscle under both metabolically disturbed untreated and metabolically controlled hyperinsulinized situations [1].

A better comprehension of the role of Slc2a4 expression in IR was gained when transgenic mice models were examined. At a glance, Slc2a4 knockout mice develop IR [9], and, conversely, Slc2a 4 overexpression improves glycemic control of diabetic mice [10]. These data reinforce the fundamental role of SLC2A4 expression on glycemic homeostasis.

In summary, increasing SLC2A4 expression would be desirable as a pharmacological treatment for IR/DM as much as decreasing SLC2A4 expression would be a valuable preventive approach against obesity.

\section{SLC2A4 pharmacogenetic bases}

Except for a study in an Indian population in which the rs5435 SNP was associated with T2DM [11], and a previous genome-wide scan performed in caucasians that found the region harboring the SLC2A4 gene as associated with metabolic syndrome [12], variants in the SLC2A4 gene have been uncommonly associated with T2DM or obesity [13-15]. However, controlling SLC2A4 expression in target tissues, which can be modulated by genetic variants, is a promising intervention to treat these conditions.

Because genes involved in glucose metabolism were described as over-represented among genes undergoing positive selection during human evolution [16], SCL2A4 diversity was investigated by sequencing SLC2A4 and genotyping 104 SNPs along an approximately $1 \mathrm{Mb}$ region flanking this gene in ethnically diverse individuals (Africans, Europeans, Asians and Latin Americans) [17]. A total of 29 polymorphisms were detected. Located in the N-terminal region, upstream of exon 7, eight SNPs presenting a minor allele frequency $(\mathrm{MAF})>5 \%$ in at least one population were identified (rs5415, rs5417 and rs5418 [5'-UTR]; rs222847, rs222849 and rs16956647 [intron 1]; rs5435 [exon 4] and rs5436 [intron 6]), along with another 15 SNPs with MAF $<5 \%$. On the other hand, the C-terminal region, downstream of intron 6 , harbored only six SNPs with MAF $<5 \%$. The pattern of genetic variation in the $\mathrm{N}$-terminal region (with common and rare variants) is compatible with neutral evolution, but the absence of common variants in 
the $\mathrm{C}$-terminal region is compatible with natural selection decreasing the degree of substitution in this region [17]. The functional implications of these findings are unknown at the moment, but they might be relevant for comprehending SLC2A4 gene-expression control.

\section{“...increasing SLC2A4 expression would be desirable as a pharmacological treatment for insulin resistance/diabetes mellitus as much as decreasing SLC2A4 expression would be a valuable preventive approach against obesity."}

To the best of the authors' knowledge, the only SLC2A4 SNP that has been functionally investigated is rs5418 (G/A); the A allele eliciting a higher luciferase activity in a gene reporter assay than the $\mathrm{G}$ allele and being overrepresented in top-level long distance Chinese runners $(61.8 \%)$ in comparison to healthy controls (45.6\%) [18]. Interestingly, it was shown that healthy Japanese men who are homozygotes for the A allele of rs 5418 presented significant lower HbAlc concentrations than AG+GG carriers [19]. Should these data be confirmed, one might ask whether T2DM carriers of the A allele could present a better metabolic response to exercise or even other therapeutic interventions known to increase SCL2A4 expression, such as glitazones.

The synonymous rs5435 SNP, located in the SCL2A4 coding region and associated with T2DM in Indian patients [11], did not influence expression of SCL2A4 in muscle tissues of T2DM patients [20], but its relation with GLUT4 expression in adipose tissue was not evaluated. As is frequently the case in studies of genetic variants in polygenic conditions, the mechanisms underlying the association observed between rs 5435 and T2DM is not known but could include changes in protein folding, linkage disequilibrium with other SNP able to destabilize pre-mRNA or activation of cryptic splicing sites, among others [11].
The knowledge accumulated so far concerning SCL2A4 transcriptional regulation leads us to believe that pharmacogenomics has the potential to be applied to select subjects more or less prone to respond to therapeutic interventions that modulate GLUT4 expression. However, the future establishment of personalized insulin sensitizer therapy will require much more effort to further understand SCL2A4 biology.

\section{Summary}

The plain expression of the SLC2A4 gene, guaranteeing effective GLUT4 protein expression and the consequent insulin-induced glucose uptake by skeletal muscle and adipose tissue, plays a fundamental role on glycemic control. Insulin resistant condition, a feature of diabetes, is known to involve decreased SLC2A4 expression and to augment its expression, has a powerful insulinsensitizer effect. This makes the SLC2A4 gene an important target for the pharmacogenomics of IR. Although variants in the $S L C 2 A 4$ gene were not associated with diabetes and obesity in the past, recent studies have detected several SNPs in its flanking region. For instance, the rs 5435 SNP was associated with T2DM, and the rs 5418 SNP was associated with skeletal muscle performance and HbAlc levels. Thus, it is clear that pharmacogenomics has great potential to select subjects more or less prone to regulate the $S L C 2 A 4$ gene and consequently glycemic homeostasis.

\section{Financial \& competing interests disclosure}

The authors acknowledge grants from FAPESP (Fundação de Amparo à Pesquisa do Estado de São Paulo), which have supported referenced studies from our research group in recent decades. The authors have no other relevant affiliations or financial involvement with any organization or entity with a financial interest in or financial conflict with the subject matter or materials discussed in the manuscript apart from those disclosed.

No writing assistance was utilized in the production of this manuscript.

\section{References}

1 Okamoto MM, Anhê GF, Sabino-Silva R et al. Intensive insulin treatment induces insulin resistance in diabetic rats by impairing glucose metabolism-related mechanisms in muscle and liver. J. Endocrinol. 211(1), 55-64 (2011).

2 Machado UF, Shimizu I, Saito M. Reduced content and preserved translocation of glucose transporter (GLUT 4) in white adipose tissue of obese mice. Physiol. Behav. 55(4), 621-625 (1994).
3 Marsh BJ, Martin S, Melvin DR et al. Mutational analysis of the carboxy-terminal phosphorylation site of GLUT-4 in 3T3-L1 adipocytes. Am. J. Physiol. 275(3), E412-E422 (1998).

4 Zaarour N, Berenguer M, Le Marchand-Brustel Y, Govers R. Deciphering the role of GLUT4 $N$-glycosylation in adipocyte and muscle cell models. Biochem. J. 445(2), 265-273 (2012).

5 Herman MA, Kahn BB. Glucose transport and sensing in the maintenance of glucose homeostasis and metabolic harmony. J. Clin. Invest. 116(7), 1767-1775 (2006).
6 Seraphim PM, Nunes MT, Machado UF. GLUT4 protein expression in obese and lean 12-month-old rats: insights from different types of data analysis. Braz. J. Med. Biol. Res. 34(10), 1353-1362 (2001).

7 Machado UF, Shimizu Y, Saito M. Decreased glucose transporter (GLUT 4) content in insulin-sensitive tissues of obese aurothioglucose- and monosodium glutamatetreated mice. Horm. Metab. Res. 25(9), 462-465 (1993).

8 de Carvalho Papa P, Vargas AM, da Silva JL, Nunes MT, Machado UF. GLUT4 protein is 
differently modulated during development of obesity in monosodium glutamate-treated mice. Life Sci. 71(16), 1917-1928 (2002).

9 Zisman A, Peroni OD, Abel ED et al. Targeted disruption of the glucose transporter 4 selectively in muscle causes insulin resistance and glucose intolerance. Nat. Med. 6(8), $924-9928$ (2000).

10 Gibbs EM, Stock JL, McCoid SC et al. Glycemic improvement in diabetic $\mathrm{db} / \mathrm{db}$ mice by overexpression of the human insulinregulatable glucose transporter (GLUT4). J. Clin. Invest. 95(4), 1512-1518 (1995).

11 Bodhini D, Radha V, Ghosh S, Majumder PP, Rao MR, Mohan V. GLUT4 gene polymorphisms and their association with Type 2 diabetes in south Indians. Diabetes Technol. Ther. 13(9), 913-920 (2011).

12 Kissebah AH, Sonnenberg GE, Myklebust J et al. Quantitative trait loci on chromosomes 3 and 17 influence phenotypes of the metabolic syndrome. Proc. Natl Acad. Sci. USA 97(26), 14478-14483 (2000).
13 Choi WH, O'Rahilly S, Buse JB et al. Molecular scanning of insulin-responsive glucose transporter (GLUT4) gene in NIDDM subjects. Diabetes 40(12), 1712-1718 (1991).

14 Lesage S, Zouali H, Vionnet N et al. Genetic analyses of glucose transporter genes in French non-insulin-dependent diabetic families. Diabetes Metab. 23(2), 137-142 (1997).

15 Friedel S, Antwerpen B, Hoch A et al. Glucose transporter 4 gene: association studies pertaining to alleles of two polymorphisms in extremely obese children and adolescents and in normal and underweight controls. Ann. NY Acad. Sci. 967, 554-557 (2002).

16 Haygood R, Fedrigo O, Hanson B, Yokoyama KD, Wray GA. Promoter regions of many neural- and nutrition-related genes have experienced positive selection during human evolution. Nat. Genet. 39(9), 1140-1144 (2007).
17 Tarazona-Santos E, Fabbri C, Yeager M et al. Diversity in the glucose transporter- 4 gene (SLC2A4) in humans reflects the action of natural selection along the old-world primates evolution. PLoS ONE 5(3), 1-10 (2010).

18 Xia X, Hu Y, Xu L, Chen T. A functional promoter polymorphism of $S L C 2 A 4$ is associated with aerobic endurance in a Chinese population. Eur. J. Sport Sci. 2012, 1-7 (2012).

19 Xi C, Miyaki K, Ikeda S, Song Y, Sinbo T, Muramatsu M. Association of GLUT4 gene variants with $\mathrm{HbAlc}$ level in Japanese men. Endocr. J. 59(8), 677-684 (2012).

20 Bjørbaek C, Echwald SM, Hubricht P et al. Genetic variants in promoters and coding regions of the muscle glycogen synthase and the insulin-responsive GLUT4 genes in NIDDM. Diabetes 43(8), 976-983 (1994). 